\title{
Publisher Correction: Semi-solid alkali metal electrodes enabling high critical current densities in solid electrolyte batteries
}

Richard J.-Y. Park, Christopher M. Eschler (D), Cole D. Fincher, Andres F. Badel (D), Pinwen Guan, Matt Pharr (D), Brian W. Sheldon (D), W. Craig Carter (D), Venkatasubramanian Viswanathan (D) and Yet-Ming Chiang (D)

Correction to: Nature Energy https://doi.org/10.1038/s41560-021-00786-w, published online 15 March 2021.

In the version of this Article originally published, in Fig. $5 \mathrm{~b}$ legend, in the label for the light blue left-facing triangle '0.95K' was incorrect and should have been ' $0.90 \mathrm{~K}$ '; this has now been corrected in all versions of the Article.

Published online: 31 March 2021

https://doi.org/10.1038/s41560-021-00819-4

๑ The Author(s), under exclusive licence to Springer Nature Limited 2021 